\title{
INFLUÊNCIA DE SISTEMAS DE CULTURAS, MUCUNA PRETAE ADUBAÇÃO MINERAL SOBRE A QUALIDADE FISIOLÓGICA DE SEMENTES DE MILHO ${ }^{1}$
}

\author{
HELBER VERAS NUNES ${ }^{2}$, IVANDRO DE FRANÇADA SILVA ${ }^{3}$, RISELANE DE LUCENAALCÂNTARABRUNO4, DANIELLAINÁCIO BARROS², \\ WALTERESFRAINPEREIRA ${ }^{5}$
}

\begin{abstract}
RESUMO - O cultivo do milho em consórcio e a utilização de leguminosas como forma de adubação é prática bastante utilizada pelo agricultor nordestino. Desta forma, o presente trabalho teve como objetivo avaliar a qualidade fisiológica das sementes de milho, cv. BR-106, produzidas em sistemas de culturas isolados e consorciados (milho, milho + feijão guandu e milho + fava), na ausência e presença de adubo químico e mucuna preta. O ensaio foi conduzido no Laboratório de Análise de Sementes da Universidade Federal da Paraíba - Campus II, Areia - PB. Independentemente dos sistemas de culturas, adubo mineral e mucuna preta, a germinação, a emergência de plântulas em areia e o índice de velocidade de emergência foram altos. De acordo com os resultados, observa-se que $\mathrm{o}$ adubo mineral favorece a viabilidade e o vigor das sementes de milho; a mucuna preta além de não competir com os sistemas de culturas favorece a qualidade fisiológica das sementes de milho provenientes do consórcio e o consórcio de milho + guandu favorece o vigor das sementes de milho.
\end{abstract}

Termos para indexação: Zea mays, consórcio, viabilidade, vigor.

\section{CULTURE SYSTEMS, VELVET BEANAND MINERAL FERTILIZATION INFLUENCE ON MAIZE SEEDS PHYSIOLOGICAL QUALITY}

\begin{abstract}
Intercropping maize cultivation and the legume species use as a fertilization method is a quite common procedure used by brazilian Northeastern farmer. Thus, this work had as an objective to evaluate maize seeds physiological quality (Zea mays L.), cv. BR-106, produced in isolated and intercropped culture systems (maize, maize + guandu bean and maize + faba bean in the absence and presence of chemical fertilizer and velvet bean. The essay was lead at the Seeds Analysis Laboratory of Paraíba Federal University - Campus II, Areia - PB. Independently of the culture systems, mineral fertilizer and velvet bean, germination, seedlings emergency in sand and the emergency speed index were high. From the results, it is observed that the mineral fertilizer favored maize seeds viability and vigor; velvet bean, besides not having competed with the culture systems, favored the physiological quality of maize seeds coming from intercropping and the intercropping maize + guandu bean favored the vigor the seed maize.
\end{abstract}

Index terms: Zea mays, intercropping, viability, vigor.

\section{INTRODUÇÃO}

O cultivo do milho em consórcio vem sendo bastante utilizado pelo agricultor nordestino por favorecer tanto na sua alimentação quanto na renda da família, além de amenizar os problemas relacionados às irregularidades climáticas, ficando

\footnotetext{
${ }^{1}$ Submetido em 15/08/2005. Aceito para publicação em 04/04/2006. Parte da tese de doutorado do primeiro autor;

${ }^{2}$ Eng. Agrônomo, Doutorando do Programa de Pós-Graduação em Agronomia, Departamento de Fitotecnia, CCA/UFPB, Areia-PB, Bolsistas CNPq. helberveras@yahoo.com.br; danyinacio@yahoo.com.br;

${ }^{3}$ Professor Adjunto, Dr., Programa de Pós-Graduação em Agronomia,
}

menos exposto às perdas totais de produção. A mucuna preta (Styzolobium aterrimum Piper et Tracy), utilizada como adubo verde, freqüentemente tem sido semeada em consórcio com o milho (Myasaka et al., 1984; Scherer e Baldissera, 1988). Costa (1992), avaliando a influência dos sistemas de cultivo sobre a qualidade fisiológica das sementes de milho e mucuna

Departamento de Solos e Engenharia Rural do CCA/UFPB, Areia - PB;

${ }^{4}$ Professor Adjunto, Dr., Programa de Pós-Graduação em Agronomia, Departamento de Fitotecnia, CCA/UFPB, Areia - PB, Bolsista CNPq. lane@cca.ufpb.com.br;

${ }^{5}$ Eng. Agrônomo, Professor, Dr., Programa de Pós-Graduação em Agronomia, Departamento de Ciências Fundamentais e Sociais do CCA/UFPB, Areia PB. 
preta, observou que houve influência dos sistemas de cultivo sobre a qualidade fisiológica das sementes de mucuna. Piana (1994) observou que a deficiência hídrica retarda o desenvolvimento das plântulas durante a germinação das sementes de milho, sendo que as mais vigorosas apresentaram desempenho superior nessa condição. Segundo Tonin (1997), a resistência ao estresse hídrico está diretamente relacionada ao vigor das sementes de milho. Sementes mais vigorosas asseguram maiores velocidades e porcentagens de germinação e influenciam, portanto, no estande e no arranjo espacial das culturas (Ellis, 1992). Aguero et al. (1997) relatam que as diferenças na qualidade fisiológica das sementes de soja, devem-se principalmente aos efeitos inerentes do ambiente durante a fase de maturação.

É desejado que as sementes utilizadas em lavouras provenham de campos isentos de outras espécies ou variedades para que assegurar a pureza varietal e outros atributos de qualidade (Carvalho e Nakagawa, 2000). Todavia, na agricultura Paraibana, caracterizada pelo predomínio de culturas de subsistência e pela pouca disponibilidade de recursos, somado ao elevado custo de sementes certificadas, verifica-se que os agricultores têm utilizado como semente seu próprio material produzido. Diante deste quadro, a avaliação dos sistemas agrícolas disponíveis é imprescindível para que possa ser introduzida melhoria tecnológica acessível aos agricultores, devendo nesta avaliação incluir a qualidade fisiológica do material produzido para ser utilizado como semente.

A avaliação da qualidade fisiológica das sementes é fundamental para os diversos segmentos que compõem um sistema de produção, pois a descoberta dos efeitos dos fatores que possam afetar a qualidade dessas sementes, depende diretamente da eficiência dos métodos utilizados para determiná-la (Marcos Filho et al., 1987). As alterações na qualidade das sementes têm como conseqüências finais a redução na capacidade germinativa, entretanto, transformações degenerativas mais sutis, não avaliadas pelo teste de germinação, exercem grande influência no potencial de desempenho, com reflexos na emergência das plântulas no campo, no crescimento e na produtividade das plantas (Spinola et al., 2000). Assim, os testes de vigor são parâmetros fundamentais para detectar essas informações e, conseqüentemente, úteis na escolha da semente a ser utilizada (Vanzolini e Nakagawa, 1998).

Considerando, a conjuntura predominante da agricultura na região Nordeste, a importância do milho e as possibilidades de melhorar a tecnologia predominante utilizada, o presente trabalho foi realizado com o objetivo de avaliar a qualidade fisiológica das sementes de milho, produzidas em sistemas de culturas isolados e consorciados, na ausência e presença de adubação verde (mucuna preta) e mineral.

\section{MATERIAL E MÉTODOS}

Este trabalho foi conduzido, em Nitossolo Vermelho, cujo as principais características são: $\mathrm{pH}=5,85$; matéria orgânica $=17,15 \mathrm{~g} \cdot \mathrm{dm}^{-3} ;$ fósforo $=2,79 \mathrm{mg} \cdot \mathrm{dm}^{-3} ;$ potássio $=$ $27,35 \mathrm{cmol}_{\mathrm{c}} \cdot \mathrm{dm}^{-3}$ e cálcio + magnésio $=9,3 \mathrm{cmol}_{\mathrm{c}} \cdot \mathrm{dm}^{-3}$, localizado no município de Alagoinha - PB, microrregião de Guarabira, tendo como coordenadas geográficas latitude Sul entre $6^{\circ} 54^{\prime} 16^{\prime \prime}$ e $6^{\circ} 59^{\prime} 44^{\prime \prime}$ e Longitude sul entre $35^{\circ} 27^{\prime} 57^{\prime \prime}$ e $35^{\circ} 36^{\prime} 00^{\prime \prime}$ e altitude de $140 \mathrm{~m}$, cujo clima de acordo com a classificação de Köppen, é do tipo As', caracterizado por ser quente e úmido com precipitação pluvial anual em torno de $1.100 \mathrm{~mm}$.

O experimento seguiu o delineamento experimental de blocos ao acaso, com parcelas subdivididas e três repetições. As parcelas principais $(5 \times 16 \mathrm{~m})$ consistiram de três sistemas de culturas \{milho (Zea mays), cultivar BR-106 em monocultivo; milho + feijão guandu (Cajanus cajan); milho + fava (Phaseolus lunatus) $\}$, plantadas no início do período chuvoso (março de 2003$)$ e as subparcelas $(5 \times 4 m)$ de duas doses de adubação mineral $\left\{0 \mathrm{~kg} \cdot \mathrm{ha}^{-1}\right.$ e $825 \mathrm{~kg} \cdot \mathrm{ha}^{-1}$ da formulação 90-60-45\}, aplicados em faixas. A adubação verde (ausência e presença), na forma de mucuna preta (Styzolobium aterrimum), foi realizado em faixas nos blocos (subblocos com $15 \times 8 \mathrm{~m}$ ). O espaçamento utilizado no milho foi $1,0 \times 0,20 \mathrm{~m}$ (50.000plantas.ha ${ }^{-1}$ ), o feijão guandu plantado intercalado entre fileiras alternadas de milho, semeado em covas espaçadas de 0,50m (10.000plantas/ha), a fava semeada em fileiras alternadas de milho em covas espaçadas de $1,0 \mathrm{~m}$, duas plantas por cova (10.000plantas.ha $\left.{ }^{-1}\right)$ e a mucuna preta semeada intercalada entre fileiras de milho, 85 dias após o plantio dos sistemas, em covas espaçadas de 0,25m (40.000plantas.ha $\left.{ }^{-1}\right)$. O manejo das plantas de cobertura do solo foi realizado antes do plantio dos sistemas, através de corte manual e distribuição sobre a superfície do solo.

Após a colheita, as sementes de milho foram misturadas de acordo com seus respectivos blocos, retiradas amostras e acondicionadas em embalagens de polietileno com $0,07 \mathrm{~mm}$ de espessura e capacidade para $15 \mathrm{~kg}$, mantidos por período de três meses em câmara fria e seca com temperatura e umidade relativa variando de $14-16^{\circ} \mathrm{C}$ e $60-70 \%$, respectivamente. As amostras foram encaminhadas ao 
Laboratório de Análise de Sementes, do Centro de Ciências Agrárias, Universidade Federal da Paraíba em Areia para a avaliação da qualidade fisiológica, sendo submetidas aos seguintes testes:

Germinação - realizado com quatro subamostras de 50 sementes distribuídas em folhas de papel "germitest", umedecidas com água destilada em quantidade equivalente a 2,5 vezes o peso do substrato seco (Brasil, 1992), sendo os rolos confeccionados e mantidos em germinador a $25^{\circ} \mathrm{C}$. As avaliações foram realizadas aos quatro e sete dias após a semeadura e os resultados expressos em porcentagem de plântulas normais.

Primeira contagem de germinação - conduzido simultaneamente ao teste de germinação, constituiu no registro da porcentagem de plântulas normais obtida no quarto dia após a semeadura.

Condutividade elétrica - utilizaram-se quatro subamostras de 50 sementes, pesadas em balança analítica de precisão de $(0,001 \mathrm{~g})$, colocadas para embeber em copos plásticos contendo $75 \mathrm{~mL}$ de água destilada, mantidos a $25^{\circ} \mathrm{C}$ durante 24 horas, conforme metodologia de Vieira (1994). Os resultados foram expressos em $\mu \mathrm{S} . \mathrm{cm}^{-1} \cdot \mathrm{g}^{-1}$ de sementes.

Emergência de plântulas em areia - conduzido em casa-de-vegetação utilizando-se bandejas plásticas contendo areia esterilizada, onde foram semeadas quatro subamostras de 50 sementes, umedecidas com a quantidade de água equivalente a $60 \%$ da capacidade de retenção. A contagem das plântulas emergidas foi realizada aos 14 dias após a semeadura (Nakagawa, 1994), sendo os resultados expressos em porcentagem.

Velocidade de emergência - realizado conjuntamente com a emergência de plântulas em areia. Foram feitas contagens diárias a partir do dia em que a primeira plântula emergiu até alcançar número constante. O índice foi calculado de acordo com a fórmula proposta por Edmond e Drapala (1958).

Massa seca da parte aérea de plântulas - a parte aérea das plântulas foram colocadas em sacos de papel, levados para estufa com circulação de ar forçado, mantidos a temperatura de $65^{\circ} \mathrm{C}$, onde permaneceram até atingir peso constante. Os resultados foram expressos em mg.plântula ${ }^{-1}$, conforme recomendações de Nakagawa (1994).

Frio sem solo - utilizaram-se quatro subamostras de 50 sementes, semeadas em folhas papel "germitest" umedecidas com água destilada, em quantidade equivalente a 2,5 vezes o peso do substrato seco. Os rolos de papel com as sementes de milho foram acondicionados em sacos plásticos, vedados com fita crepe e, posteriormente, colocados em câmara regulada a $10^{\circ} \mathrm{C}$ onde permaneceram por 7 dias. Após esse período, os sacos foram abertos e os rolos colocados em germinador a $25^{\circ} \mathrm{C}$, durante quatro dias (Cícero e Vieira, 1994). Os resultados foram expressos em porcentagem de plântulas normais.

Frio com solo - foram adotados os mesmos procedimentos do teste anterior, distribuindo-se sobre as sementes uma fina camada de solo recentemente cultivado com milho.

Procedimento Estatístico - os tratamentos foram distribuídos conforme o delineamento experimental inteiramente casualizado, em laboratório e em casa-devegetação. Os dados foram submetidos à análise de variância, sendo as médias comparadas por meio de contrastes ortogonais.

\section{RESULTADOS E DISCUSSÃO}

Os valores médios de germinação, primeira contagem de germinação, emergência de plântulas em areia, condutividade elétrica, velocidade de emergência, massa seca da parte aérea, teste frio com solo e frio sem solo de sementes de milho estão apresentados na Figura 1. Independentemente dos sistemas de culturas, adubo mineral e mucuna preta, a germinação, emergência de plântulas em areia e o índice de velocidade de emergência foram altos, variando de 85,0 a $97,0 \% ; 85,0$ a $100 \%$ e 17,0 a 22,0, respectivamente, indicando que, de modo geral, as sementes apresentavam boa viabilidade, enquadrando-se acima dos padrões mínimos exigidos (85\%) para comercialização de sementes de milho (Brasil, 1993).

O teste de germinação (Tabela 1) indicou que as sementes de milho, provenientes das subparcelas com adubo mineral e dos cultivos consorciados, na presença da mucuna preta, adubada ou não, tiveram maior porcentagem de germinação (17,3; 3,0 e 2,5\%), em relação às produzidas em monocultivo, não havendo diferença entre os sistemas consorciados. De acordo com Malavolta e Romero (1975), o milho é bastante exigente e responsivo à adubação, com efeito sobre a planta em 70 a $90 \%$ dos ensaios conduzidos.

Estudos sobre o vigor são importantes para a agricultura, pois permitem a obtenção de estimativas do potencial fisiológico das sementes, identificando diferenças não detectadas pelo teste de geminação (Ramos et al., 2004). Para Carvalho e Nakagawa (2000), a faixa ótima de temperatura para germinação de uma semente de alto vigor é sempre mais ampla do que para uma de baixo vigor. Além disto, sementes 

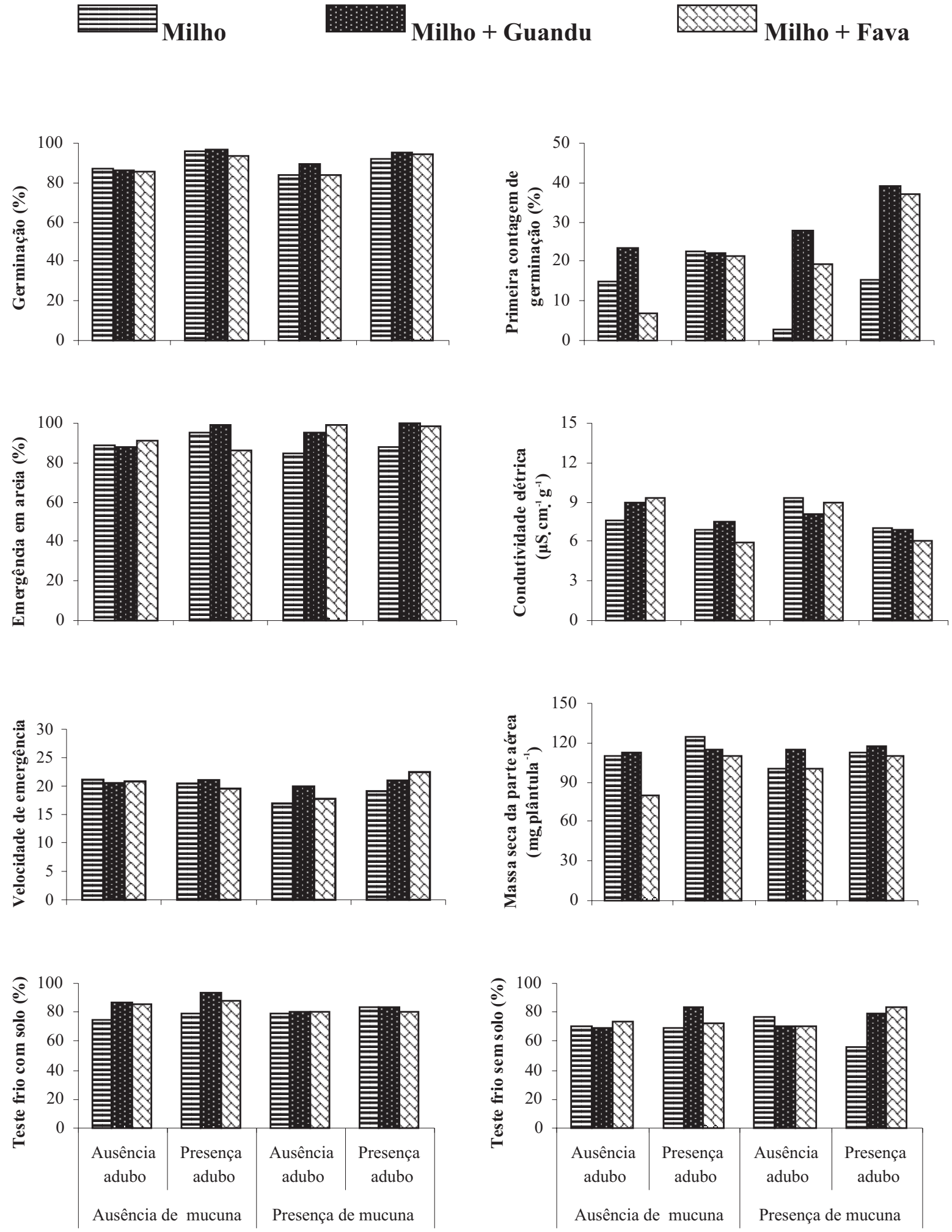

FIGURA 1. Médias dos testes de germinação, primeira contagem, emergência de plântulas em areia, condutividade elétrica, velocidade de emergência, massa seca da parte aérea, frio com solo e frio sem solo de sementes de milho, sob diferentes sistemas de culturas, mucuna preta e adubação mineral. 
TABELA 1. Estimativas dos contrastes de germinação (G), primeira contagem de germinação (PCG), emergência de plântulas em areia (EPA) e condutividade elétrica (CE) de sementes de milho, sob diferentes sistemas de culturas, mucuna preta e adubação mineral.

\begin{tabular}{lrrrr}
\hline Contrastes & $\mathrm{G}(\%)$ & $\mathrm{PCG}(\%)$ & $\mathrm{EPA}(\%)$ & $\mathrm{CE}\left(\mu \mathrm{S} \cdot \mathrm{cm}^{-1} \cdot \mathrm{g}^{-1}\right)$ \\
\hline Sem adubo (A1) vs com adubo (A2) & $-17,3^{*}$ & $-20,5^{* *}$ & $-6,5^{*}$ & $4,0^{*}$ \\
Sem mucuna (M1) vs com mucuna (M2) / A1 & $0,7^{\mathrm{ns}}$ & $-1,7^{\mathrm{ns}}$ & $-3,7^{*}$ & $-0,2^{\mathrm{ns}}$ \\
M1 vs M2 / A2 & $1,3^{\mathrm{ns}}$ & $-8,5^{\mathrm{ns}}$ & $-1,8^{\mathrm{ns}}$ & $0,2^{\mathrm{ns}}$ \\
Solteiro vs consórcio / M1A1 & $1,0^{\mathrm{ns}}$ & $-0,3^{\mathrm{ns}}$ & $-0,5^{\mathrm{ns}}$ & $-1,5^{\mathrm{ns}}$ \\
Solteiro vs consórcio / M1A2 & $1,0^{\mathrm{ns}}$ & $0,8^{\mathrm{ns}}$ & $3,0^{\mathrm{ns}}$ & $0,2^{\mathrm{ns}}$ \\
Solteiro vs consórcio / M2A1 & $-2,5^{* *}$ & $-20,7^{* *}$ & $-12,0^{* *}$ & $0,8^{\mathrm{ns}}$ \\
Solteiro vs consórcio / M2A2 & $-3,0^{* *}$ & $-22,5^{* *}$ & $-11,0^{* *}$ & $0,6^{\mathrm{ns}}$ \\
Milho + guandu vs milho + fava / M1A1 & $1,0^{\mathrm{ns}}$ & $16,5^{* *}$ & $-3,0^{\mathrm{ns}}$ & $-0,4^{\mathrm{ns}}$ \\
Milho + guandu vs milho + fava / M1A2 & $3,0^{\mathrm{ns}}$ & $0,5^{\mathrm{ns}}$ & $13,0^{* *}$ & $1,6^{\mathrm{ns}}$ \\
Milho + guandu vs milho + fava / M2A1 & $6,0^{\mathrm{ns}}$ & $8,5^{\mathrm{ns}}$ & $-4,0^{\mathrm{ns}}$ & $-0,9^{\mathrm{ns}}$ \\
Milho + guandu vs milho + fava / M2A2 & $1,0^{\mathrm{ns}}$ & $2,0^{\mathrm{ns}}$ & $2,0^{\mathrm{ns}}$ & $0,9^{\mathrm{ns}}$ \\
\hline
\end{tabular}

ns, * e **. Não significativo, significativo a 5 e $1 \%$ de probabilidade, respectivamente, pelo teste $\mathrm{F}$.

mais vigorosas são mais resistentes às condições de estresse hídrico (Tekrony e Egli, 1977). Pelos resultados das Tabelas 1 e 2, observa-se que os testes não foram igualmente sensíveis para distinguir diferenças no vigor das sementes. Assim, como o teste de germinação, a primeira contagem indicou que o adubo mineral e o cultivo consorciado, na presença da mucuna preta, adubada ou não, aumentou o vigor das sementes (20,5; 22,5 e $20,7 \%$ ) em relação às produzidas em monocultivo; todavia, sementes provenientes do consórcio milho + guandu foram mais vigorosas $(16,5 \%)$ em relação ao consórcio milho + fava, entretanto com o incremento do adubo mineral ou da mucuna esta diferença desaparece. Tais diferenças provavelmente devem-se a utilização de leguminosas em consórcio ao disponibilizar mais nitrogênio proporcionado pela fixação biológica (Teixeira et al., 1994); ao feijão guandu por ser leguminosa de sistema radicular profundo e eficiente na ciclagem de nutrientes e à fava ser uma cultura extratora. Apesar da existência na literatura de resultados de pesquisa que indicam o efeito benéfico da adubação verde, esta prática continua restrita a reduzido número de agricultores.

Os resultados referentes à emergência das plântulas em areia demonstraram comportamento semelhante ao da primeira contagem de germinação, indicando, de maneira geral, efeito benéfico do adubo e da mucuna preta (6,5 e 3,7\%). A mucuna preta, adubada ou não, favoreceu a emergência das plântulas $(11,0$ e $12,0 \%)$ das sementes de milho provenientes dos cultivos consorciados em relação às produzidas em monocultivo. Além de apresentar crescimento rápido e possuir grande quantidade de massa verde para cobertura do solo, algumas espécies de mucuna podem evitar a multiplicação de nematóides fitoparasitas que causam grandes danos às culturas principais (Chaves e Calegari, 2001). Nunes et al. (2004), estudando a qualidade sanitária de sementes de milho procedentes de plantio direto, verificaram que a mucuna preta favoreceu o controle de Penicillium sp. Entre os consórcios, apesar das sementes apresentarem comportamento semelhantes, somente na presença do adubo mineral, o consórcio milho + guandu foi mais vigoroso (13,0\%). Vários trabalhos evidenciam que sementes com baixo vigor apresentam baixa emergência de plântulas (Edje e Burris, 1971; Tekrony e Egli, 1977; Marcos Filho, 1981). De acordo com Pádua (1988), sementes pouco vigorosas possuem menor capacidade de estabelecimento em condições de campo.

Os valores de condutividade elétrica (Figura 1) das sementes de milho foram baixos $\left(6\right.$ a $\left.9 \mu \mathrm{S}_{\mathrm{cm}} \mathrm{cm}^{-1} \cdot \mathrm{g}^{-1}\right)$, com pequena lixiviação de solutos, confirmando sua alta qualidade fisiológica. Verifica-se que a adubação mineral aumentou o vigor das sementes de milho (Tabela 1). Todavia, a condutividade elétrica e o índice de velocidade de emergência (Tabela 2) não se mostraram testes sensíveis para boa diferenciação do vigor.

A massa seca da parte aérea de plântulas de milho (Tabela 2) sofreu incremento tanto na adição do adubo mineral (24,0mg.plântula $\left.{ }^{-1}\right)$ quanto para as sementes provenientes do consórcio milho + guandu (32,5mg.plântula $\left.{ }^{-1}\right)$ em relação ao consórcio milho + fava $\left(15,0 \mathrm{mg}\right.$.plântula $\left.\mathrm{a}^{-1}\right)$, entretanto com o incremento do adubo mineral ou da mucuna esta diferença desapareceu. De acordo com Nakagawa (1994), sementes mais vigorosas proporcionam maior transferência de matéria seca de seus tecidos de reserva para o eixo embrionário na 
TABELA2. Estimativas dos contrastes da velocidade de emergência (VE), massa seca da parte aérea de plântulas de milho (MSPA), teste frio com solo (FCS) e frio sem solo (FSS) de sementes de milho, sob diferentes sistemas de culturas e adubações

\begin{tabular}{|c|c|c|c|c|}
\hline Contrastes & $\mathrm{VE}$ & $\begin{array}{c}\text { MSPA } \\
\text { (mg.plântula }^{-1} \text { ) }\end{array}$ & $\begin{array}{l}\text { FCS } \\
(\%)\end{array}$ & $\begin{array}{l}\text { FSS } \\
(\%)\end{array}$ \\
\hline Sem adubo (A1) vs com adubo (A2) & $-2,2^{n s}$ & $-24,0 *$ & $-6,5^{n s}$ & $-4,7^{\mathrm{ns}}$ \\
\hline Sem mucuna (M1) vs com mucuna (M2) / A1 & $2,6^{\mathrm{ns}}$ & $-4,2^{n s}$ & $2,2^{\mathrm{ns}}$ & $-1,0^{\mathrm{ns}}$ \\
\hline $\mathrm{M} 1 \mathrm{vs} \mathrm{M} 2$ / A2 & $-0,5^{n s}$ & $3,5^{\mathrm{ns}}$ & $4,2^{\mathrm{ns}}$ & $2,0^{\mathrm{ns}}$ \\
\hline Solteiro vs consórcio / M1A1 & $0,6^{\mathrm{ns}}$ & $13,8^{\mathrm{ns}}$ & $-10,9 * *$ & $-1,0^{\mathrm{ns}}$ \\
\hline Solteiro vs consórcio / M1A2 & $0,2^{\mathrm{ns}}$ & $12,5^{\mathrm{ns}}$ & $-11,3 * *$ & $-9,0^{\mathrm{ns}}$ \\
\hline Solteiro vs consórcio / M2A1 & $-1,9^{n s}$ & $-7,5^{\mathrm{ns}}$ & $-0,8^{\mathrm{ns}}$ & $6,6^{\mathrm{ns}}$ \\
\hline Solteiro vs consórcio / M2A2 & $-2,6^{\mathrm{ns}}$ & $-1,0^{\mathrm{ns}}$ & $1,1^{\mathrm{ns}}$ & $-26,3 * *$ \\
\hline Milho + guandu vs milho + fava / M1A1 & $-0,3^{n s}$ & $32,5 * *$ & $1,1^{\mathrm{ns}}$ & $-4,0^{\mathrm{ns}}$ \\
\hline Milho + guandu vs milho + fava / M1A2 & $1,6^{\mathrm{ns}}$ & $5,0^{\mathrm{ns}}$ & $5,5^{\mathrm{ns}}$ & $11,0 *$ \\
\hline Milho + guandu vs milho + fava / M2A1 & $2,2^{\mathrm{ns}}$ & $15,0^{\mathrm{ns}}$ & $-0,5$ ns & $0,0^{\text {ns }}$ \\
\hline Milho + guandu vs milho + fava / M2A2 & $-1,5^{\mathrm{ns}}$ & $7,0^{\mathrm{ns}}$ & 2,8 ns & $-4,5^{\mathrm{ns}}$ \\
\hline
\end{tabular}

ns ${ }^{*}$ e $* *$. Não significativo, significativo a 5 e $1 \%$ de probabilidade, respectivamente, pelo teste $\mathrm{F}$.

fase de germinação, originando plântulas com maior peso em função do maior acúmulo de matéria seca em suas partes.

Para os testes de frio com e sem solo (Tabela 2) as sementes de milho provenientes do consórcio mostraram-se, de maneira geral, mais vigorosas pelas razões comentadas anteriormente. Já a mucuna preta semeada, aproximadamente, 85 dias após a introdução dos sistemas não foi prejudicial às sementes de milho, conforme verificado em todos os testes utilizados.

\section{CONCLUSÕES}

$\mathrm{O}$ adubo mineral favorece a viabilidade e o vigor das sementes de milho.

A mucuna preta além de não competir com os sistemas de culturas favorece a qualidade fisiológica das sementes de milho provenientes do consórcio.

O consórcio de milho e feijão guandu favorece o vigor da semente de milho.

\section{REFERÊNCIAS}

AGUERO, J.A.P.; VIEIRA, R.D.; BITTENCOURT, S.R. Avaliação da qualidade fisiológica da sementes de cultivares de soja. Revista Brasileira de Sementes, Brasília, v.19, n.2, p.255-260, 1997.

BRASIL. Ministério da Agricultura e Reforma Agrária. Regras para análise de sementes. Brasília: SNDA/DNPV/CLAV, 1992.365p.

BRASIL. Ministério da Agricultura, do Abastecimento e da Reforma Agrária. Padrões estaduais de sementes. Brasília: EMBRAPA/SPSB, 1993.47p.
CARVAlHo, N.M.; NAKAGAWA, J. Sementes: ciência, tecnologia e produção. Jaboticabal: FUNEP, 2000. 588p.

CHAVES, J.C.D.; CALEGARI, A. Informe Agropecuário, Belo Horizonte, v.22, p.53-60, 2001.

CÍCERO, S.M.; VIEIRA, R.D. Teste de frio. In: VIEIRA, R.D.; CARVALHO, N.M. Testes de vigor em sementes. Jaboticabal: FUNEP, p.151-164, 1994.

COSTA, R.F. Influência dos sistemas de cultivo e do beneficiamento sobre a qualidade das sementes de milho (Zea mays L.) armazenadas. 1992. 82f. Dissertação (Mestrado em Produção Vegetal) - Universidade Federal da Paraíba, Centro de Ciências Agrárias, Areia, 1992.

EDMOND, J.B.; DRAPALA, W.J. The effects of temperature, sand and soil, and acetone on germination of okra seeds. Procedings of American Society for Horticultural Science, Local, n.71, p.428434, 1958.

EDJE, O.T.; BURRIS, J.S. Effects of soybean seed vigor on field perfomance. Agronomy Journal, Madison, v.63, p.536-538, 1971.

ELLIS, R.H. Seed and seedling vigor in relation to crop growth and yield. Plant Grow Regulator, v.11, n.3, p.249-255, 1992.

MALAVOLTA, E.; ROMERO, J.P. Manual de adubação. 2 ed. São Paulo: ANDA. 1975. 349p.

MARCOS FILHO, J.; CÍCERO, S.M.; SILVA, W.R. Avaliação da qualidade das sementes. Piracicaba: ESALQ/USP, 1987. 230p.

MARCOS FILHO, J. Qualidade fisiológica e maturação de sementes de soja cultivares Bragg e UFV - 1 e comportamento das plantas no campo. Pesquisa Agropecuária Brasileira, Brasília, v.16, n.3, p.405-415, 1981.

MYASAKA, S.; CAMARGO, D.A.; CAVALIERI, P.A. Adubação orgânica, adubação verde e rotação de culturas no estado de São Paulo. São Paulo: Cargill, 1984. 138p.

NAKAGAWA, J. Testes de vigor baseados na avaliação das 
plântulas. In: VIEIRA, R.D.; CARVALHO, N.M. Testes de vigor em sementes. Jaboticabal: FUNEP, p.49-85, 1994.

NUNES, H.V.; BARROS, D.I.; SILVA, I.F.; ARAÚJO, E.; SOUTO, F.M. Influência de diferentes sistemas de culturas e adubações sobre a qualidade sanitária de sementes de milho em plantio direto. In: Simpósio Brasileiro de Patologia de Sementes, 8, João Pessoa - PB. 2004. Anais..., UFPB, 2004. p.202.

PÁDUA, G.P. Vigor de sementes e seus possíveis efeitos sobre a emergência em campo e a produtividade. Informativo ABRATES, Londrina, v.8, n.1/2/3, p.46-49,1998.

PIANA, Z. Respostas de sementes de milho, com diferentes níveis de vigor, à disponibilidade hídrica. 1994. 107f. Tese (Doutorado em Agronomia) - Escola Superior de Agricultura "Luiz de Queiroz"/ USP, Piracicaba, 1994.

RAMOS, P.N.; FLOR, E.P.O; MENDONÇA, E.A.F.; MINAMI, K. Envelhecimento acelerado em sementes de rúcula (Eruca sativa L.). Revista Brasileira de Sementes, Pelotas, v.26, n.1, p.98-103, 2004.

SCHERER, E.C.; BALDISSERA, I.J. Mucuna: a proteção do solo em lavoura de milho. Agropecuária Catarinense, Florianópolis, v.1, n.1, p.21-25, 1985.
SPINOLA, M.C.M.; CÍCERO, S.M.; MELO, M. Alterações bioquímicas e fisiológicas em sementes de milho causadas pelo envelhecimento acelerado. Scientia Agrícola, Piracicaba, v.57, n.2, p.263-270, 2000.

TEIXEIRA, L.A.J.; TESTA, V.M.; MIELCZUK, J. Nitrogênio no solo, nutrição e rendimento de milho afetados por sistemas de cultura. Revista Brasileira Ciência do Solo, Campinas, v.18, n.2, p.207-214, 1994.

TEKRONY, D.M.; EGLI, D.B. Relationship between laboratory indices of soybean seed vigor and field emergence. Crop Science, Madison, v.17, p.573-577, 1977.

TONIN, G.A. Influência do genótipo e do vigor sobre o desempenho germinativo de sementes de milho (Zea mays L.) sob condições de estresse hídrico. 1997. 54f. Dissertação (Mestrado em Agronomia) - FCAV/Universidade Estadual Paulista, Jaboticabal, 1997.

VANZOLINI, S.; NAKAGAWA, J. Teste de condutividade elétrica em genótipos de sementes de amendoim. Revista Brasileira de Sementes, Brasília, v.20, n.1, p.178-183, 1998.

VIEIRA, R.D. Teste de condutividade elétrica. In: VIEIRA, R.D.; CARVALHO, N.M. Testes de vigor em sementes. Jaboticabal: FUNEP, p.103-132, 1994. 